\title{
AUTOMORPHISMS OF CAYLEY GRAPHS OF METACYCLIC GROUPS OF PRIME-POWER ORDER
}

\author{
CAI HENG LI and HYO-SEOB SIM
}

To Laci Kovács on his 65 th birthday

(Received 17 October 2000)

Communicated by R. A. Bryce

\begin{abstract}
This paper investigates the automorphism groups of Cayley graphs of metacyclic $p$-groups. A characterization is given of the automorphism groups of Cayley graphs of a metacyclic $p$-group for odd prime $p$. In particular, a complete determination of the automorphism group of a connected Cayley graph with valency less than $2 p$ of a nonabelian metacyclic $p$-group is obtained as a consequence. In subsequent work, the result of this paper has been applied to solve several problems in graph theory.
\end{abstract}

2000 Mathematics subject classification: primary $05 \mathrm{C} 25,20 \mathrm{~B} 25$.

\section{Introduction}

Let $G$ be a finite group, and let $S$ be a subset of $G$ that does not contain the identity 1 of $G$. If $S=S^{-1}:=\left\{s^{-1} \mid s \in S\right\}$, the graph with the vertex set $G$ and the edge set $\{\{x, s x\} \mid x \in G, s \in S\}$ is called a Cayley graph of $G$ and denoted by $\operatorname{Cay}(G, S)$.

The adjacency relation of the graph $\operatorname{Cay}(G, S)$ is uniquely determined by the group $G$ and the subset $S$, and so are some simple properties of Cay $(G, S)$, for example, Cay $(G, S)$ is a regular graph with valency $|S|$, and $\operatorname{Cay}(G, S)$ is connected if and only if $\langle S\rangle=G$. However, to understand some further graph structure properties of the graph, for example, how symmetric the graph is, we often need to know the full

The second author was supported by a grant No. KOSEF 96-0701-03-01-3 from Korea Science and Engineering Foundation.

(C) 2001 Australian Mathematical Society 0263-6115/2001 \$A2.00+0.00 
automorphism group of $\operatorname{Cay}(G, S)$. By the definition, it is easy to see that the group $G$ acts regularly on the vertex set $G$ by right multiplication (that is, $g$ acts on $x$ as the product $x g$ ) and so $G$ may be viewed as a regular subgroup of the automorphism group of the Cayley graph. In particular, the automorphism group of a Cayley graph acts transitively on the vertex set. But in general the problem of determining the full automorphism group of a Cayley graph is very difficult. Since a Cayley graph $\Gamma=\operatorname{Cay}(G, S)$ is defined by $G$, a natural approach to the problem is to understand the relationship between the full automorphism group Aut $\Gamma$ and $G$, for example, whether or not $G$, as a regular subgroup, is normal in Aut $\Gamma$.

For convenience, a Cayley graph $\Gamma$ will be called normal if the regular subgroup $G$ is normal in Aut $\Gamma$ (see [21]). The automorphism group of a normal Cayley graph $\Gamma=\operatorname{Cay}(G, S)$ is a semidirect product of the regular normal subgroup $G$ by the subgroup $\operatorname{Aut}(G, S)$ which consists of all automorphisms of the group $G$ that fix $S$ setwise (see Lemma 2.1). The automorphisms of the graph $\Gamma$ are therefore completely determined by automorphisms of the group $G$. Usually, the latter is much easier to be determined. Thus a natural problem is to determine normality of Cayley graphs for a given class of groups.

The problem determining normality of Cayley graphs of a given cyclic group of prime order was solved by Alspach [1]; some partial answers for other classes of groups to this problem can be found in several papers, for example $[3,6,11,20]$. The main purpose of the paper is to characterize the automorphism groups of certain Cayley graphs for metacyclic groups of prime-power order, in view of normality.

For two groups $G$ and $H$, let $G \rtimes H$ be a semidirect product of $G$ by $H$. For a subset $S$ of a group $G$, write

$$
\operatorname{Aut}(G, S):=\{\theta \in \operatorname{Aut}(G) \mid \theta(S)=S\} .
$$

The first result of this paper determines automorphism groups of Cayley graphs of a nonabelian metacyclic $p$-group in the case when $p$ is an odd prime that does not divide the order of $\operatorname{Aut}(G, S)$.

THEOREM 1.1. Let $G$ be a finite nonabelian metacyclic $p$-group for an odd prime $p$, and let $\Gamma=\operatorname{Cay}(G, S)$ be a Cayley graph of $G$. Assume that Aut $(G, S)$ is a $p^{\prime}$-group. Then either Aut $\Gamma \cong G \rtimes \operatorname{Aut}(G, S)$, or $G \cong \mathbb{Z}_{9} \rtimes \mathbb{Z}_{3^{\prime}}$ and $\operatorname{Aut} \Gamma \cong \operatorname{PSL}(2,8) \rtimes \mathbb{Z}_{3^{\prime}}$, where $r \geq 1$.

Note that the $p^{\prime}$-group $\operatorname{Aut}(G, S)$ in the theorem is a cyclic group of order dividing $p-1$ (see $[14,15])$. We then have a complete determination of the automorphism group of a connected Cayley graph with valency less than $2 p$ for a nonabelian metacyclic $p$-group of odd order. 
COROLLARY 1.2. Let $\Gamma=\operatorname{Cay}(G, S)$ be a connected Cayley graph with valency less than $2 p$ of a finite nonabelian metacyclic $p$-group $G$ for an odd prime $p$. Then Aut $\Gamma \cong G \rtimes \operatorname{Aut}(G, S)$.

One of the motivations of studying normal Cayley graphs comes from some problems of graph theory. A graph is said to be half-transitive if it is vertex-transitive and edge-transitive but not arc-transitive. Initiated with a question of Tutte [19, page 60], half-transitive graphs have received considerable attention for many years (see, for example, $[2,4,16,20])$. In [13], the result of this paper has been applied to construct and characterize an interesting class of half-transitive graphs. A Cayley graph $\Gamma$ of a group $G$ is called a graphical regular representation of $G$ if Aut $\Gamma=G$. The problem of deciding whether a Cayley graph is a graphical regular representation of the corresponding group is a long-standing one, see [7]. The result given in Theorem 1.1 is used in [12] to solve the problem for metacyclic $p$-groups.

After we describe some background results in Section 2, we will prove Theorem 1.1 and Corollary 1.2 in Section 3.

\section{Background results}

We first setup some notation and terminology. Let $G$ be a group. Denote by $\Phi(G)$ the Frattini subgroup of $G$. The product of all minimal normal subgroups of $G$ is called the socle of $G$ and is denoted by $\operatorname{Soc}(G)$. The automorphism group and the outer automorphism group of $G$ are denoted by $\operatorname{Aut}(G)$ and $\operatorname{Out}(G)$, respectively. For two subgroups $H$ and $K$ of $G$, let $\mathbf{C}_{H}(K)$ denote the centralizer of $K$ in $H$, and let $\mathbf{N}_{H}(K)$ denote the normalizer of $K$ in $H$.

We now collect some basic results, which will be used in this paper.

LEMMA 2.1. Let $\Gamma=\operatorname{Cay}(G, S)$ be a Cayley graph of a finite group $G$. Then $\mathbf{N}_{\text {Aut } \Gamma}(G)=G \rtimes \operatorname{Aut}(G, S)$.

Proof. Write $A:=$ Aut $\Gamma$. The normalizer of $G$ in the symmetric group $\operatorname{Sym}(G)$ is $G \times \operatorname{Aut}(G)$ (see [5, Corollary 4.2B]). So we have

$$
\mathbf{N}_{A}(G)=(G \rtimes \operatorname{Aut}(G)) \cap A=G \rtimes(\operatorname{Aut}(G) \cap A) .
$$

Obviously, $\operatorname{Aut}(G) \cap A=\operatorname{Aut}(G, S)$.

We also note that a proof of this lemma may be found in [7, Lemma 2.1].

Lemma 2.2. Let $\Gamma=\operatorname{Cay}(G, S)$ be a Cayley graph of a finite $p$-group $G$. If $\operatorname{Aut}(G, S)$ is a $p^{\prime}$-group, then $G$, viewed as a regular subgroup, is a Sylow p-subgroup of Aut $\Gamma$. 
Proof. Write $A=$ Aut $\Gamma$. Suppose that $\operatorname{Aut}(G, S)$ is a $p^{\prime}$-group. Then $\mathbf{N}_{A}(G) / G$ is a $p^{\prime}$-group. If $G$ is not a Sylow $p$-subgroup of $A$, then $G$ is a proper subgroup of a Sylow $p$-subgroup $P$ of $A$. Thus $G<\mathbf{N}_{P}(G) \leq \mathbf{N}_{A}(G)$ (see [17, page 88]), which is a contradiction since $\mathbf{N}_{A}(G) / G$ is a $p^{\prime}$-group.

We also need the following facts about finite simple groups with a subgroup of prime-power index. First we prove a property about outer automorphisms and Schur multipliers of such simple groups.

LEMMA 2.3. Let $p$ be an odd prime. Let $T$ be a nonabelian simple group which has a subgroup $H$ of index $p^{l}>1$, and let $M(T)$ be the Schur multiplier of $T$. Then

(i) $p \nmid|M(T)|$;

(ii) either $p \nmid|\operatorname{Out}(T)|$ or $T \cong \operatorname{PSL}(2,8)$ and $p^{l}=3^{2}$.

PROOF. The finite nonabelian simple groups $T$ with a subgroup $H$ of prime-power index were classified by Guralnick in [9], and the Schur Multipliers of finite simple groups are completely classified, see Table 4.1 in [8, page 302]. Combining these two classifications, we only need to check the case that $T=\operatorname{PSL}(n, q)$ and $\left(q^{n}-1\right) /(q-1)=p^{l}$, where $q=r^{f}$ for some prime $r$ and some positive integer $f$. It is known that

$$
|M(T)|=d, \quad|\operatorname{Out}(T)|= \begin{cases}2 d f & \text { if } n \geq 3, \\ d f & \text { if } n=2,\end{cases}
$$

where $d=\operatorname{gcd}(n, q-1)$. If $n f \leq 2$, then $T=\operatorname{PSL}(2, r)$ and $|\operatorname{Out}(T)|=2$, so $p \nmid|\operatorname{Out}(T)|$ and $p \nmid|M(T)|$. Assume that $n f \geq 3$. If $r=2$ and $n f=6$, then it follows that $\left(q^{n}-1\right) /(q-1)=3^{2}$ and so $T=\operatorname{PSL}(2,8)$ and $|M(T)|=1$ in this case. If $(r, n f) \neq(2,6)$ then by Zigmondy Theorem (see [10, IX 8.3 and 8.4]), there is a (primitive) prime $k>n f$ such that $k \mid\left(r^{n f}-1\right)$ but $k \nmid\left(r^{f}-1\right)$. Thus $k \mid\left(q^{n}-1\right) /(q-1)=p^{l}$ and so $k=p$. In particular, $p>d f$, and hence $p \nmid|\operatorname{Out}(T)|$ and $p \nmid|M(T)|$.

The following lemma is an immediate consequence of Corollary 2 in Guralnick [9].

LEMMA 2.4. Let $T$ be a nonabelian simple group acting transitively on $\Omega$ with $p^{l}$ elements for a prime $p$. If $p$ does not divide the order of a point-stabilizer in $T$, then $T$ acts 2-transitively on $\Omega$.

Finally, we observe a fact on transitive permutation groups of prime-power degree.

LEMMA 2.5. Let $p$ be a prime, and let $A$ be a transitive permutation group on $\Omega$ of prime-power degree. Let $B$ be a nontrivial subnormal subgroup of $A$. Then $B$ has a proper subgroup of $p$-power index, and $\mathbf{O}_{p^{\prime}}(B)=1$. In particular, $\mathbf{O}_{p^{\prime}}(A)=1$. 
PROOF. The assumption that $B$ is subnormal in $A$ means that there exists a series of subgroups $B \unlhd B_{1} \unlhd \cdots \unlhd B_{k}=A$. Let $v$ be a point in $\Omega$ which is not fixed by $B$. Since $A$ is transitive on $\Omega, B_{k-1}$ is half-transitive on $\Omega$. Thus the $B_{k-1}$-orbit $O_{k-1}$ containing $v$ is of $p$-power size. Similarly, $B_{k-2}$ is half-transitive on $O_{k-1}$, and thus the $B_{k-2}$-orbit $O_{k-2}$ containing $v$ is also of $p$-power size. Repeating this argument, we have that the $B$-orbit containing $v$ is of $p$-power size, and so $B$ has a proper subgroup of $p$-power index. So the subnormal subgroup $\mathbf{O}_{p^{\prime}}(B)$ has a subgroup of $p$-power index, and hence $\mathbf{O}_{p^{\prime}}(B)=1$. In particular, taking $B=A$, we have that $\mathbf{O}_{p^{\prime}}(A)=1$.

\section{Proofs of the main results}

In this section, we prove the main results, that is, Theorem 1.1 and Corollary 1.2. We will proceed the proofs with a series of lemmas. We recall that a metacyclic group is a group $G$ which has a cyclic normal subgroup $K$ such that $G / K$ is cyclic. We notice that every subgroup and every quotient group of a metacyclic group are also metacyclic, and in particular, can be generated by at most two elements.

Let $G$ be a finite nonabelian metacyclic $p$-group for an odd prime $p$, and let $\Gamma=\operatorname{Cay}(G, S)$ be a Cayley graph of $G$. Let $A$ denote the automorphism group of the Cayley graph $\Gamma$, and let $A_{1}$ denote the group of all automorphisms of $\Gamma$ that fix the identity 1 of $G$. To prove Theorem 1.1, we assume that $\operatorname{Aut}(G, S)$ is a $p^{\prime}$-group. Then by Lemma 2.2, $G$ is a Sylow $p$-subgroups of Aut $\Gamma$, or equivalently, $p$ does not divide $\left|A_{1}\right|$.

\section{LEMMA 3.1. The graph $\Gamma$ is not a complete graph.}

Proof. Suppose that $\Gamma$ is a complete graph, that is, $\Gamma=\mathrm{K}_{n}$, where $n=|G|$. Then $A=\mathrm{S}_{n}$, the symmetric group of degree $n$. However, a Sylow $p$-subgroup of $\mathrm{S}_{n}$ is not isomorphic to the nonabelian metacyclic group $G$, which is a contradiction.

LEMMA 3.2. If $N$ be a nonabelian minimal normal subgroup of $A$, then $p=3$ and $N \cong \operatorname{PSL}(2,8)$.

PROOF. Assume that $N$ is non-abelian minimal normal subgroup. Then $N=$ $T_{1} \times \cdots \times T_{k}$, where $T_{i} \cong T$ for some nonabelian simple group $T$. By Lemma $2.5,|N|$ is divisible by $p$. The normal subgroup $N$ has a Sylow $p$-subgroup contained in $G$. Since $G$ is metacyclic, it follows that $k$ is at most 2. By Lemma 2.5, $T_{1}$ has a subgroup of $p$-power index. Thus by Lemma 2.3 (ii), either $p=3$ and $T_{1}=\operatorname{PSL}(2,8)$ or $p$ does not divide $\left|\operatorname{Out}\left(T_{1}\right)\right|$. 
Assume first that $p$ does not divide $\left|\operatorname{Out}\left(T_{1}\right)\right|$. Since $\mathbf{N}_{A}\left(T_{1}\right) / T_{1} \mathbf{C}_{A}\left(T_{1}\right)$ is isomorphic to a subgroup of $\operatorname{Out}\left(T_{1}\right)$, it follows that $|G|$ divides $\left|T_{1} \mathrm{C}_{A}\left(T_{1}\right)\right|$. Since $T_{1}$ is nonabelian simple, $T_{1} \cap \mathbf{C}_{A}\left(T_{1}\right)=1$, and hence the product $T_{1} \mathbf{C}_{A}\left(T_{1}\right)$ is a direct product. Suppose that $p \nmid\left|\mathbf{C}_{A}\left(T_{1}\right)\right|$. Then $T_{1}$ contains a Sylow $p$-subgroup of $A$, and hence $T_{1}$ is transitive on the vertex set $G$. By Lemma 2.4, noting that $p$ does not divide $\left|A_{1}\right|, T_{1}$ is 2-transitive on the vertex set $G$. So $\Gamma$ is a complete graph, which contradicts Lemma 3.1. Therefore, $p$ divides $\left|\mathbf{C}_{A}\left(T_{1}\right)\right|$. Taking a Sylow $p$-subgroup $P_{1}$ of $T_{1}$ and a Sylow $p$-subgroup $P_{2}$ of $\mathrm{C}_{A}\left(T_{1}\right)$, we see that $G$ is conjugate to $P_{1} \times P_{2}$. Consequently, the $P_{i}$ are cyclic, and so $G$ is abelian, which is not the case.

Assume now that $p=3$ and $T_{1}=\operatorname{PSL}(2,8)$. Consider the case where $k=2$, namely $N=T_{1} \times T_{2}$. As $N \cap \mathbf{C}_{A}(N)=1,\left\langle N, \mathbf{C}_{A}(N)\right\rangle=N \times \mathbf{C}_{A}(N)$. By Lemma 2.5 , if $\mathbf{C}_{A}(N) \neq 1$ then 3 divides $\left|\mathbf{C}_{A}(N)\right|$, and thus a Sylow 3-subgroup of $N \times \mathbf{C}_{A}(N)$ is isomorphic to $\mathbb{Z}_{9} \times \mathbb{Z}_{9} \times P$ for some nontrivial 3-group $P$, which is a contradiction since $G$ is metacyclic. Hence $\mathbf{C}_{A}(N)=1$. Write $B=\mathbf{N}_{A}\left(T_{1}\right)$. Then $B=\mathbf{N}_{A}\left(T_{2}\right)$ and $B$ is a normal subgroup of $A$ with index 2. Both of $\mathbf{C}_{A}\left(T_{1}\right)$ and $\mathrm{C}_{A}\left(T_{2}\right)$ are also normal in $B$. Since $\mathbf{C}_{A}\left(T_{1}\right) \cap \mathbf{C}_{A}\left(T_{2}\right)=\mathbf{C}_{A}(N)=1$, it follows that $B$ is isomorphic to a (subdirect) subgroup of $B / C_{A}\left(T_{1}\right) \times B / C_{A}\left(T_{2}\right)$, and so $B$ is isomorphic to a subgroup of $\operatorname{Aut}\left(T_{1}\right) \times \operatorname{Aut}\left(T_{2}\right)$. Let $Q$ be a Sylow 3-subgroup of $B$. Then $Q$ is also a Sylow 3-subgroup of $A$ and $G \cap N$ is a normal subgroup of $G$ isomorphic to $\mathbb{Z}_{9} \times \mathbb{Z}_{9}$. Since $G$ is nonabelian metacyclic, $G$ has an element of order 27; however, there is no such an element in $\operatorname{Aut}(\operatorname{PSL}(2,8))$, and so no such an element in $\operatorname{Aut}\left(T_{1}\right) \times \operatorname{Aut}\left(T_{2}\right)$, a contradiction. Therefore, $k=1$ and $N \cong \operatorname{PSL}(2,8)$.

We then have a consequence of Lemma 3.2.

LEMMA 3.3. Either $\operatorname{Soc}(A)$ is soluble or $G=\mathbb{Z}_{9} \rtimes \mathbb{Z}_{3^{\prime}}$ and $A=\operatorname{PSL}(2,8) \rtimes \mathbb{Z}_{3^{\prime}}$, where $r \geq 1$.

Proof. Suppose that $\operatorname{Soc}(A)$ is insoluble. Then by Lemma 3.2, $p=3$ and $A$ has a minimal normal subgroup $N$ such that $N \cong \operatorname{PSL}(2,8)$. Let $C=\mathrm{C}_{A}(N)$. Then $A / C$ is isomorphic to a subgroup of $\operatorname{Aut}(N) \cong N \rtimes \mathbb{Z}_{3}$. As $G$ is nonabelian metacyclic, it follows that $A / C \cong N \rtimes \mathbb{Z}_{3}$. So $A / C=L / C \rtimes B / C$, where $L / C \cong N$ and $B / C \cong \mathbb{Z}_{3}$. Since $L \cap N$ is normal in the simple group $N$, we see that $N \leq L$, and so $N \cap B=1$. Thus $A=N \rtimes B$. Let $P$ be a Sylow 3-subgroup of $B$. Then $P$ is cyclic and $B=C P$. Let $M$ be the normalizer of $P$ in $B$. Then $M=(C \cap M) P$. Since $P /(C \cap P) \cong B / C \cong \mathbb{Z}_{3}$, we have $C \cap M \geq C \cap P=\Phi(P)$, the Frattini subgroup of $P$, and hence $M / \Phi(P)=(C \cap M) / \Phi(P) \times P / \Phi(P)$. So the subgroup $C \cap M$ acts trivially on $P / \Phi(P)$, which implies that $C \cap M$ acts trivially on $P$ also. Thus $P$ centralizes the normalizer $M$ of the Sylow 3-subgroup $P$. It then follows from Burnside's Theorem for $p$-nilpotency that $B$ is 3-nilpotent. Thus the normal Hall 
3 -subgroup of $B$ is a characteristic subgroup of $C$ and so it is a normal $p^{\prime}$-subgroup of $A$. By Lemma 2.5 , we have $B=P$. Therefore, $A=N \rtimes P$, as desired.

We will also prove the following lemmas.

LEMMA 3.4. If $\operatorname{Soc}(A)$ is soluble, then $\mathbf{C}_{A}\left(\mathbf{O}_{p}(A)\right) \leq \mathbf{O}_{p}(A)$.

PROOF. Suppose that $B$ is a normal semisimple subgroup of $A$. From the definition of a semisimple group, we see that $B=B^{\prime}$, and $B / Z(B)$ is a direct product of nonabelian simple groups. By Lemma $2.5, B$ has a subgroup of $p$-power index, and in particular, $B / Z(B)$ has a subgroup of $p$-power index. It follows from Lemma 2.5 that $\mathbf{Z}(B)$ is a $p$-group. Since $B / \mathbf{Z}(B)$ is a direct product of nonabelian simple groups, we see from Lemma 2.3(i) that $p \nmid|M(B / Z(B))|$. So $\mathbf{Z}(B)=1$. Thus $B$ is a direct product of nonabelian simple groups, and so $B$ contains an insoluble minimal normal subgroup of $A$. This yields a contradiction to the assumption. Thus $A$ has no normal semisimple subgroups. By the definition (see [18, Definition 6.10, page 452]), the generalized Fitting subgroup $\mathbf{F}^{*}(A)$ equals the Fitting subgroup $\mathbf{F}(A)$. By Lemma 2.5 , $\mathbf{O}_{p^{\prime}}(A)=1$, and thus $\mathbf{F}^{*}(A)=\mathbf{F}(A)=\mathbf{O}_{p}(A)$. Therefore, $\mathbf{C}_{A}\left(\mathbf{O}_{p}(A)\right) \leq \mathbf{O}_{p}(A)$. The lemma follows from Lemma 2.5.

LEMMA 3.5. If $\operatorname{Soc}(A)$ is soluble then $G=\mathbf{O}_{p}(A) \unlhd A$.

Proof. Let $H=\mathbf{O}_{p}(A)$. It follows from Lemma 3.4 that $\mathbf{C}_{A}(H) \leq H$. Write $V=H / \Phi(H)$ and $\bar{A}=A / \Phi(H)$. Then $V$ may be regarded as a vector space over $\mathbb{Z}_{p}$. We consider the action of $A$ on $V$ by conjugation. Since $H$ acts trivially on $V$, we have $H \leq \mathbf{C}_{A}(V)$ and $\mathbf{C}_{A}(V)$ is normal in $A$. Suppose that $H$ is a proper subgroup of $\mathrm{C}_{A}(V)$. Then $\mathbf{C}_{A}(V)$ has a nontrivial $p^{\prime}$-element $x$. Since the $p^{\prime}$-element $x$ acts trivially on $H / \Phi(H)$, we see that $x$ acts also trivially on $H$. So $x \in \mathbf{C}_{A}(H)$ but $x$ is not contained in $H$. This yields a contradiction since $\mathbf{C}_{A}(H) \leq H$. Therefore $\mathbf{C}_{A}(V)=H$, and so the conjugation leads a faithful representation of $A / H$ as a subgroup of GL(V).

If $V \cong \mathbb{Z}_{p}$, then $A / H$ is isomorphic to a subgroup of a cyclic group of order $p-1$; in this case $H$ is the Sylow $p$-subgroup of $A$ and so $G=\mathbf{O}_{p}(A)$. We now consider the remaining case, namely when $V \cong \mathbb{Z}_{p} \times \mathbb{Z}_{p}$. Suppose that $H<G$. Then $A / H$ is isomorphic to a subgroup $L$ of $\operatorname{GL}(2, p)$. Since $H<G$, a Sylow $p$-subgroup of $L$ is not normal. Then by [17, Theorem 6.17, page 404], $L \cap \operatorname{SL}(2, p)$ contains $\operatorname{SL}(2, p)$, and hence $\operatorname{SL}(2, p) \leq L$. Since $1<\mathbf{Z}(\operatorname{SL}(2, p)) \leq \mathbf{O}_{p^{\prime}}(L)$ for odd $p$, we see that $1<\mathbf{O}_{p^{\prime}}(L)$. We also have $\mathbf{O}_{p, p^{\prime}}(\bar{A})=V \rtimes Q$, where $Q \cong \mathbf{O}_{p^{\prime}}(L)$. Since $\operatorname{SL}(2, p) \leq L, V$ is a minimal normal subgroup of $\bar{A}$. It follows from $\mathbf{Z}\left(\mathbf{O}_{p, p^{\prime}}(\bar{A})\right)=\mathbf{C}_{V}(Q) \times \mathbf{C}_{Q}(V)=\mathbf{C}_{V}(Q)$ that $\mathbf{C}_{V}(Q)$ is normal in $\bar{A}$. Therefore $\mathbf{C}_{V}(Q)=1$. Further, by the Frattini argument (or see [17, (8.12), page 238], 
$\bar{A}=V \mathbf{N}_{\bar{A}}(Q)$. Since $V \cap \mathbf{N}_{\bar{A}}(Q)=\mathbf{C}_{V}(Q)=1$, we have $\bar{A}=V \rtimes \mathbf{N}_{\bar{A}}(Q)$, and so a Sylow $p$-subgroup of $\bar{A}$ is isomorphic to $\left(\mathbb{Z}_{p} \times \mathbb{Z}_{p}\right) \rtimes \mathbb{Z}_{p}$. This is not the case since $p$ is odd and $G$ is a metacyclic $p$-group. Consequently, we have $G=\mathbf{O}_{p}(A) \unlhd A$.

PROOF OF THEOREM 1.1. To complete the proof of Theorem 1.1, we now only need to show that $A \cong G \rtimes \operatorname{Aut}(G, S)$ when $G$ is normal in Aut $\Gamma$, while it follows from Lemma 2.1. So the proof of Theorem 1.1 is now complete.

We now prove Corollary 1.2 .

ProOf OF COROLlary 1.2. Since $p$ is odd, there exists a subset $T$ of $S$ such that $T \cap T^{-1}=\emptyset, S=T \cup T^{-1}$. Since $|S|<2 p$, we have $|T|<p$. Let $\theta$ be an $p$-element in $\operatorname{Aut}(G, S)$. Assume that $\theta$ has an orbit of length $p$. Then there exists an element $t$ in $T$ such that both of $t$ and $t^{-1}$ are contained in the orbit of length $p$. This means that $t^{-1}=\theta^{k}(t)$ for some $k$ with $1 \leq k<p$. So $\left\langle\theta^{2 k}\right\rangle$ stabilizes $t$. However, $\left\langle\theta^{2 k}\right\rangle=\langle\theta\rangle$ since $p$ does not divide $2 k$. This yields a contradiction. So $\theta$ acts trivially on $S$. Since $S$ generates $G$, we see that $\theta$ acts faithfully on $S$. Thus $\theta=1$, which implies that $\operatorname{Aut}(G, S)$ is a $p^{\prime}$-group. For $p=3$, it easily follows from since $|S| \leq 4$ that the automorphism group $A$ is a $\{2,3\}$-group. So $A$ is soluble. By Theorem 1.1, we have $A=G \rtimes \operatorname{Aut}(G, S)$ for $p=3$. If $p>3$, the claim also follows from Theorem 1.1 .

\section{References}

[1] B. Alspach, 'Point-symmetric graphs and digraphs of prime order and transitive permutation groups of prime degree', J. Combin. Theory Ser. B 15 (1978), 12-17.

[2] B. Alspach and M. Y. Xu, 'Half-transitive graphs of order 3p', J. Algebraic Combin. 3 (1994), 347-355.

[3] Y. G. Baik, Y. Q. Feng, H. S. Sim and M. Y. Xu, 'On the normality of Cayley graphs of abelian groups', Algebra Colloq. 5 (1998), 287-304.

[4] I. Z. Bouwer, 'Vertex and edge-transitive but not 1-transitive graphs', Canad. Math. Bull. 13 (1970), 231-237.

[5] J. D. Dixon and B. Mortimer, Permutation groups (Springer, New York, 1996).

[6] Y.Q. Feng, The normality of Cayley digraphs with small valencies (Ph.D. Thesis, Peking University, 1997).

[7] C. D. Godsil, 'On the full automorphism group of a graph', Combinatorica 1 (1981), 243-256.

[8] D. Gorenstein, Finite simple groups (Plenum Press, New York, 1982).

[9] R. M. Guralnick, 'Subgroups of prime power index in a simple group', J. Algebra 81 (1993), 304-311.

[10] B. Huppert and N. Blackburn, Finite groups II (Springer, New York, 1982).

[11] C. H. Li, 'On isomorphisms of connected Cayley graphs III', Bull. Austral. Math. Soc. 57 (1998), 137-145. 
[12] C. H. Li and H. S. Sim, 'The graphical regular representations of finite metacyclic $p$-groups', European J. Combin. 21 (2000), 917-925.

[13] _ ' 'On half-transitive metacirulant graphs of prime-power order', J. Combin. Theory (Ser. B) 81 (2001), 45-57.

[14] W. Lindenberg, 'Die Ordnungen der Automorphismengruppen von Zerfallenden bizyklischen $p$ Gruppen', Berichte Gesellsch. Math. Datenverarb. 57 (1972), 203-215.

[15] _ - 'Eine Klasse von $p$-Gruppen, deren Automorphismengruppen $p$-Gruppen sind', Period. Math. Hungar. 5 (1974), 171-183.

[16] D. Marušič, 'Recent developments in half-transitive graphs', Discrete Math. 182 (1998), 219-231.

[17] M. Suzuki, Group theory I (Springer, New York, 1982).

[18] _ Group theory II (Springer, New York, 1986).

[19] W. T. Tutte, Connectivity in graphs (University of Toronto Press, North York, 1966).

[20] M. Y. Xu, 'Half-transitive graphs of prime-cube order', J. Algebraic Combin. 1 (1992), 275-282.

[21] _ 'Automorphism groups and isomorphisms of Cayley digraphs', Discrete Math. 182 (1998), 309-319.

Department of Mathematics and Statistics

The University of Western Australia

Nedlands WA 6907

Australia

e-mail: li@maths.uwa.edu.au
Division of Mathematical Sciences

Pukyong National University

Pusan 608-737

Korea

e-mail: hsim@pknu.ac.kr 
